Tropical Journal of Pharmaceutical Research April 2013; 12 (2): 265-273

ISSN: $1596-5996$ (print); 1596-9827 (electronic)

(C) Pharmacotherapy Group, Faculty of Pharmacy, University of Benin, Benin City, 300001 Nigeria.

All rights reserved.

Available online at http://www.tjpr.org

Review Article

http://dx.doi.org/10.4314/tjpr.v12i2.20

\title{
Effect of Zeta Potential on the Properties of Nano-Drug Delivery Systems - A Review (Part 2)
}

\author{
Soheyla Honary* and Foruhe Zahir \\ Mazandaran University of Medical Sciences, School of Pharmacy, Sari, Iran \\ *For correspondence: E-mail: shonary@yahoo.com; shonary@mazums.ac.ir \\ Received: 18 August 2012
}

Revised accepted: 7 January 2013

\begin{abstract}
The zeta potential (ZP) of colloidal systems and nano-medicines, as well as their particle size exert a major effect on the various properties of nano-drug delivery systems. Not only the stability of dosage forms and their release rate are affected but also their circulation in the blood stream and absorption into body membranes are dramatically altered by ZP. In this paper the effect of ZP on the various properties of nano-medicines are reviewed. Furthermore, the ability of employing zeta potential to target drug delivery systems to, and drug release at specific sites of the body are discussed.
\end{abstract}

Keywords: Nano-medicines, Zeta potential, Drug targeting, Site-specific release, Dosage form stability

Tropical Journal of Pharmaceutical Research is indexed by Science Citation Index (SciSearch), Scopus, International Pharmaceutical Abstract, Chemical Abstracts, Embase, Index Copernicus, EBSCO, African Index Medicus, JournalSeek, Journal Citation Reports/Science Edition, Directory of Open Access Journals (DOAJ), African Journal Online, Bioline International, Open-J-Gate and Pharmacy Abstracts

\section{INTRODUCTION}

Nanoparticle surface is a very important consideration in targeting drug delivery. Conventional nanoparticles (without surface modification) and negatively charged particles can be rapidly opsonized and massively cleared by fixed macrophages of the reticuloendothelial system (RES) in the blood stream. Surface modification of nano-drug delivery systems is the most common strategy to controlling the opsonization process and thus sustain the systems for longer period in the blood stream. Suitable ZP can improve drug release profiles at specific sites as well as their stability in dosage forms.

Effect of ZP on gene delivery systems The transfection efficiency of gene vector depends on the particle size and zeta potential [1]. A system that is simply negatively charged [2] or with a positive surface charge density might be desirable for loading DNA molecules [3].
Because many proteins, DNA and cell membrane surface are slightly anionic, so a positive ZP not only has benefits for enhanced DNA loading efficiency also might provide the effective accumulation in the target cells [4]. "Complexes" is the term in which particle formation is elicited by the tropism between DNA molecules and nanoparticles with positive surface charge [5].

The particle size and $\zeta$-potential of vector/pDNA particles can influence the intracellular trafficking of particles and subsequent transfection efficiency. The particles are large at N/P ratios of $2: 1$ to $4: 1$, corresponding to neutral or slightly positive zeta potentials, which demonstrates that particles possibly be gathered into a mass at the neutral condition. The ZP of binary complexes increases with increase in N/P ratios, and neutral or positive ZP were observed for N/P ratios of 2 and greater. The transfection efficiency of gene vector depends on the particle size and zeta potential. Moreover, excess positive charge on 
the complexes can cause the nonspecific binding and uptake by non targeted cells. Thus for complexes with targeting ligand, a weekly positively charged surface is preferable for the specific binding by receptor-mediated endocytosis. When complexes of N/P higher than the optimal value were added to the cultured cells, specific binding was inhibited in the presence of excess peptides. According to literature, arginine-rich peptides also show the same trend [6].

Previous studies aimed to develop an amphoteric hyaluronic acid (HA) derivative with polyethyleneimine (PEI) chains (HAP) for gene delivery to overcome the disadvantages of $\mathrm{PEI}$ as gene carrier including the cytotoxicity caused by excess of positive charge, non-special interaction and aggregation in the blood, and non-target gene delivery. At charge ratio of 1 , where the complex could not form completely, the zeta potential was negative. But an interesting result was observed in that the zeta potential of HAP/DNA complex with higher molecular weight $\mathrm{HA}$ at certain charge ratio was positive, but not negative charge as expected. Moreover, electrochemical equilibrium process was observed in the course of determining ZP, which signified that the condensation process of DNA by HAP might be complicate [7].

Cationic nanoparticles of biodegradable polymers such as poly (lactide) (PLA) have been shown to be promising carrier systems for DNA and siRNA delivery. The cationic poly (d,I-lactideco-glycolide) (PLGA)-PEI, PLGA-chitosan and methoxy poly (ethylene glycol)-poly (lactide) PLGA/PEl and mPEG-PLA/PEI nanoparticles were prepared by a nanoprecipitation method. Surface modification of the nanoparticles into cationic surface was done to facilitate the loading of negatively charged dsRNA. The results showed that zeta potential of the nanoparticles significantly changed from negative to positive value after being modified by the polyethyleneimine (PEI) $25 \mathrm{kDa}$ and chitosan. Since the surface charge of the nanoparticle delivery system was primarily determined by the amount of the amine group (NH2+) of PEI 25 $\mathrm{kDa}$ and the chitosan coated on the surface of the nanoparticles, four groups of nanoparticles demonstrated different zeta potential indicating different charge potential of the nanoparticles. All of the four nanoparticles showed great dsRNA loading potential. This further confirmed the previous results that nanoparticles through polycations modification can absorb the negative charge nucleic acid and load it efficiently [8] and the charge interaction is firm enough to absorb the dsRNA at the surface of nanoparticles [9].
Cationic nanoparticles combine the advantages of nanoparticles as a drug carrier and complexation ability with nucleic acid in acting as the gene carrier. With better biocompatibility and easy producing property, cationic nanoparticles have gained more and more attention in the gene delivery area. Unlike the delivery of plasmid DNA, transport of dsRNA is much easier since there is no need to deliver dsRNA into the cell nucleus (fig 1). Previous polymeric nanoparticles mediated gene delivery method tend to use double emulsion technique loading the therapeutic gene and was limited by the low encapsulation efficiency of the drug. Moreover, during the process of preparing nanoparticles through double emulsion technique, the contact of the dsRNA with a lot of organic solvent and the involvement of sonication procedure might damage or denature the dsRNA.

Now, more and more researchers have employed cationic nanoparticles simply because it is an easy, safe and efficient way to carry therapeutic substances. Chitosan and polyethyleneimine (PEI) have both been used as cationic coating agents. The results demonstrate that PEI $25 \mathrm{kDa}$ is slightly better than the chitosan by showing higher gene transfection efficiency and higher loading efficiency of RNA. These results revealed that two factors affect the siRNA delivery efficacy of cationic nanoparticles in terms of higher DNA loading capacity than chitosan [10]. In addition, the zeta potentials of poly-siRNA/PEI complexes (1.54 $\pm 0.72 \mathrm{mV})$ were lower than that of mono-siRNA/PEl complexes (22.39 $\pm 0.53 \mathrm{mV})$, implying that most of the positively charged PEI chains in polysiRNA/PEI complexes were closely covered with the strongly anionic poly-siRNA. The most positively charged portion of $\mathrm{PEI}$ in monoSiRNA/PEI complexes protruded from the complexes due to the weak electrostatic interaction with lower anionic charged monosiRNA. These results clearly reveal that polysiRNA produced more nano-sized and compact complexes than mono-siRNA via electrostatic interactions with PEI [11].

A good complexation of the negatively charged SiRNA to the cationic dex-HEMA-co-TMAEMA nanogels is a prerequisite to successfully deliver siRNA across plasma membrane into cytosol. To evaluate this, nanogel samples were titrated with siRNA and the resultant zeta-potential of the siRNA-loaded nanogels was measured. Clearly, the loading of the nanogels with siRNA is reflected in the zeta-potential value as can be seen in the typical sigmoidal curves that are obtained; the lower positive surface charge of the DS 8.9 nanogels may result in a less efficient 
cellular internalization. Fewer uptakes of DS 8.9 nanogels by Huh-7 cells could indeed be confirmed by flow cytometry and is therefore one of the reasons which explain the lower gene silencing by DS 8.9 nanogels [12].

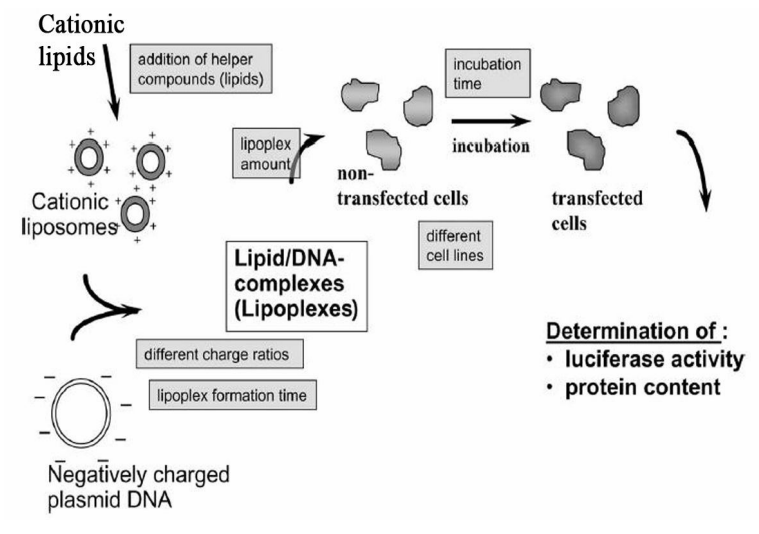

Figure 1: Formation of lipid/DNA complexes [18]

\section{Effect of ZP on macrophage uptake}

Due to large surface area/volume ratio, nanoparticles tend to agglomerate and adsorb proteins. When bound to proteins, they may be quickly cleared by macrophages before they can reach target cells [13-14]. Hence, the aggregation of serum components and the thrombus formation can also result in embolism [3].

One possible approach to increase the circulation time of nanoparticles in blood stream is to modify particle surface and minimize or eliminate protein adsorption. The mechanism of protein adsorption has been attributed to electrostatic interaction. Electrostatic interaction of the nanoparticles can be controlled by variation in their surface charges, which can be determined by measuring the zeta potential of these particles. Nanoparticles having positive zeta potential displayed good protein adsorption [14], but in one investigation by creating a highly immunogenic (opsonizable) liposome containing negatively charged lipids, these formulations were rapidly removed from circulation by the reticuloendotelial system (RES) [15]. In general, however, negatively charged samples did not significantly adsorb protein. By varying the surface charges, one can vary the electrostatic interaction between the protein and the adsorbent for selective adsorption of a particular protein.

Factors such as $\mathrm{pH}$ and solution electrolyte concentration have a considerable impact on the strengths and types of electrostatic charges on the adsorbent and thus can lead to different protein and surface interactions under different conditions [14]. The membrane of red blood cells (RBC) is also soft and composed of two-sub layers, the outer sub layer being negatively charged and the inner one being positively charged. According to some studies, the dominant factor to control the interaction between RBC and the synthetic polymer is the softness of the nanoparticle surface. It is considered that if the nanoparticles cover with synthetic polymers, they are not recognized as foreign materials by biological cells, if the polymer surfaces are soft and hydrophilic and in this way one can decrease the hemolytic potential [16].

It has been shown that NPs with high surface charge and large particle size are phagocytized more efficiently by murine macrophage. Slight particle size and surface charge differences and different cell lines had significant implications in the cellular uptake of NPs, and various mechanisms were involved in the uptake process (Fig 2). In vivo biodistribution suggested that NPs with slight negative charges and particle size of $150 \mathrm{~nm}$ were tended to accumulate in tumor more efficiently. These results could serve as a guideline in the rational design of drug nanocarriers with maximized therapeutic efficacy and predictable in vivo properties, in which the control of particle size and surface charge was of significance. It has been shown that the surface charge of rhodamine $\mathrm{B}$ (RhB) labeled carboxymethyl chitosan grafted NPs (RhBCMCNP) and chitosan hydrochloride grafted NPs (RhB-CHNP) significantly affected their uptake by phagocytic cell. Macrophage uptake increased with the surface charge increasing (either positive or negative). This process could lead to increase in phagocytic uptake up to 1.3fold. When the absolute values of Zeta potential were similar, positively charged NPs showed a higher phagocytic uptake compared to negatively charged NPs irrespective of NPs composition. The cellular uptake of FITC-labeled protamine sulfate (FITC-PS) and camptothecin (CPT) showed the same tendency with their corresponding carriers and were slightly lower. Compared to those of the same composition and particle size, NPs carrying more surface charges exhibited smaller contact angle referring to the higher hydrophilicity, which might be attributed to the thicker hydrophilic shell [17].

It is known that scavenger receptors recognize anionic particles and facilitate uptake by the RES [18]. Negatively charged spherical particles can potentially bind to available cationic sites on the macrophage surface and be recognized by scavenger receptors while adsorption of proteins on their surface may alter their overall charge, 

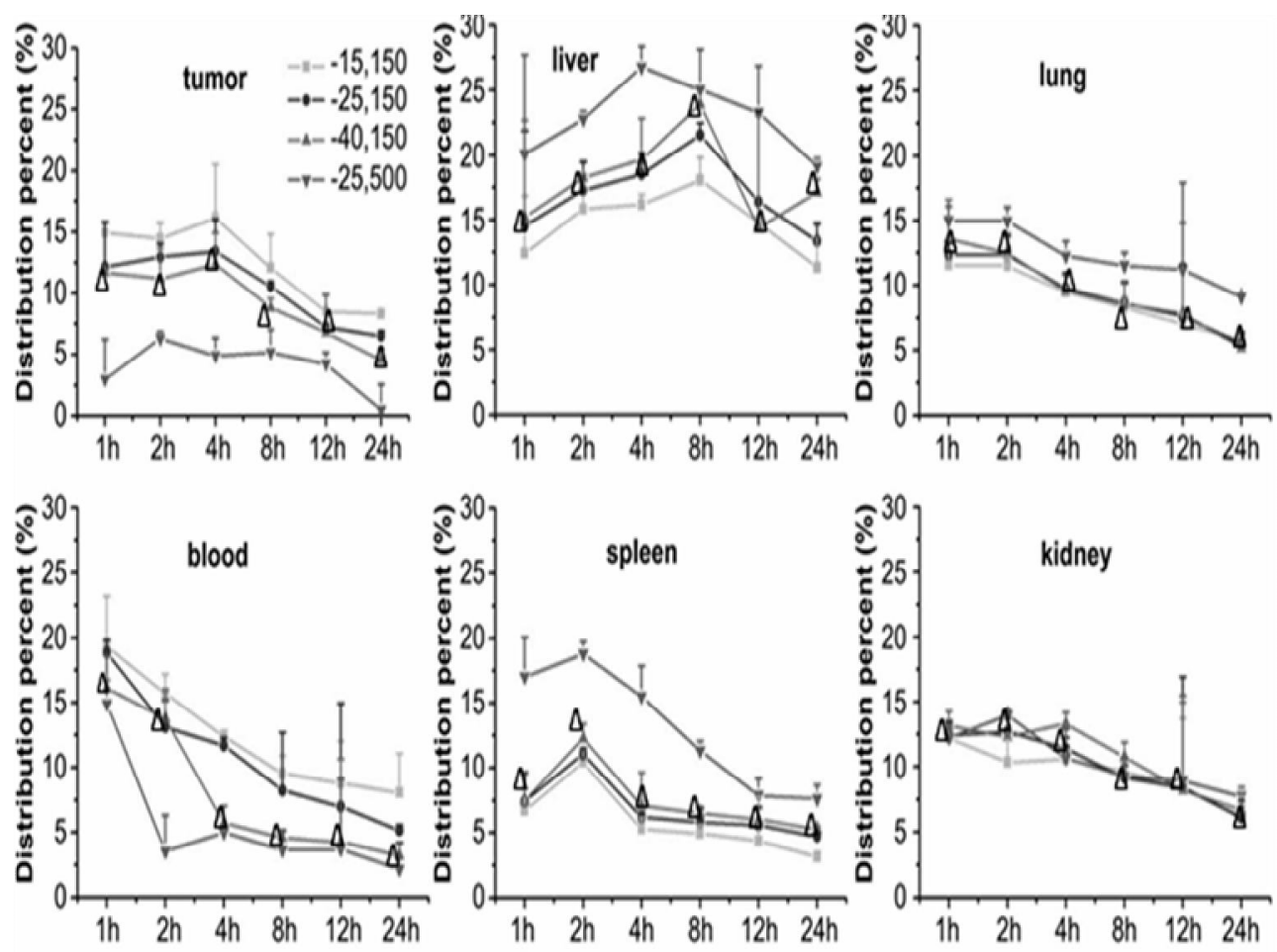

Figure 2: Biodistribution of RhB-CMCNP of varying ZP and size in different parts of the body [17]. Note: $\mathbf{a}=-15$ $\mathrm{mv}, 150 \mathrm{~nm} ; \bullet=-25 \mathrm{mv}, 150 \mathrm{~nm} ; \Delta=-40 \mathrm{~nm}, 150 \mathrm{~nm} ; \quad \nabla=-25 \mathrm{mv}, 500 \mathrm{~nm}$

prevent such interaction and result in fewer uptakes. It must be noted, however, that the zeta potential of protein-bound spherical particles is only slightly negative at $-6.1 \mathrm{mV}$, and hence in addition to the influence of surface charge, nanoparticle geometry has probably played a role in the cellular uptake and biodistribution of the nanoparticles [19].

$\mathrm{PH}-$ responsive liposomes containing synthetic glutamic acid-based zwitterionic lipids were developed by Obata et al. The zeta potential of liposomes containing 1,5-dihexadecyl Nglutamyl-L-glutamate (L1) and/or 1,5-dihexadecyl $\mathrm{N}, \mathrm{N}$-diglutamyl-lysyl-L-glutamate (L2) was positive when the solution $\mathrm{pH}$ was below 4.6 or 5.6 , respectively, but negative at higher $\mathrm{pH}$ values. The $\mathrm{pH}$-responsive liposomes showed improved fusogenic potential to an endosomemimicking anionic membrane at acidic $\mathrm{pH}$. Fusion was clearly increased when the $\mathrm{pH}$ value was less than $\mathrm{pH} 5.5$ or 6.0 for $\mathrm{L} 1$ or L2liposomes, respectively. Because the zeta potential of the L1 or L2-liposomes is positive under these $\mathrm{pH}$ conditions, the cationic liposomes should adhere to the negatively charged model membrane. Following the initial contact, membrane fusion then occurs by flip-flop movement of the lipids. Beside the fusion, the aggregation between the positively and negatively charged liposomes may occur [20].

\section{Effect of ZP on immunogenicity of nanoparticles}

There are some investigations that showed that the surface charge of nanoparticles has an effect on the stimulation of the immune response. Previous literatures mentioned that while the negatively charged liposomes were immunostimulant the zwitteronic ones were not. Other authors have observed positive results using cationic liposomes or liposomes coated with cationic polymers such as protamine [21,22].

\section{Effect of ZP on ocular drug delivery systems}

Positive ZP is important for ocular drug delivery since it can facilitate effective adhesion to the cornea epithelial surface, prolonging the drug release and enhancing the drug bioavalibility in the internal tissue of the eye due to the electrostatic interaction between the positively charged and the negatively charged present at the corneal surface [23]. Due to the highly positive charge that Chitosan Oligosaccharides (COS) carried, the adsorption of COS increased the density of positive electron cloud that resulted in the positive electricity of integral particle as well. This increment in surface charge was ascribed to the formation of complexes, with the coating mechanism involving hydrogen bonding between the polysaccharide and the glyceride head groups [24]. 
Positive charge can facilitate an effective adhesion to the corneal surface and account for a strong interaction with the negatively charged mucosa of the conjunctiva and anionic mucin present in the tear film, prolonging the residence time of the formulation [25]. It was shown that nanoplexes had a positive zeta-potential that could favour the attachment of the nanosystems onto the ocular surface. The positively charged nanoplexes interact with negatively charged sites on the cornea and tight junctions, and result in loosening or opening of the tight junctions owing to alteration in the relative concentrations of specific ion species in the pore volume. Thus, intercellular and intracellular penetration rather than simple mucoadhesion could explain the prolonged residence time (up to $24 \mathrm{~h}$ ) of the particles on the cornea [26].

\section{Effect ZP on the uptake of NPs from gut}

Factors which govern the uptake of particles from the gut include: particle size, surface charge and physicochemical proprties of the particle [27]. Investigations showed that antigen loaded cationically charged particles could be beneficial for gut up take. Cationically modified antigen is taken up more vigorously compared with non modified form [28]. The positively charged drug loaded nano particles are expected to interact with negatively charged sialic acid and fucose residues of mucin in the intestine by electrostatic interactions [29].

\section{Effect of ZP on respiratory nano-drug delivery}

Although poly(styrene) and poly(D, L-lactide-coglycolide) nanoparticles revealed a dosedependent influence on biophysics of pulmonary surfactant, positively-charged nanoparticles made from poly(butyl methacrylate-co-(2dimethylaminoethyl) methacrylate-co-methyl methacrylate) showed no effect. This behavior is attributed to differences in ZP and surface hydrophobicity, which in turn involves an altered adsorption pattern of the positively charged surfactant proteins to the nanoparticles. It was shown that adsorption of cytochrome C depended on both nanoparticle surface properties, i.e., surface charge and surface hydrophobicity [30]. Although maximal protein adsorption is usually observed with hydrophobic surfaces, a diverse effect was obtained in the case of the highly negatively-charged polymeric nanoparticles with hydrophilic surface (PLGANPs). Increasing surface hydrophilicity and keeping surface charge density almost constant led to a similar adsorption of cytochrome $\mathrm{C}$ to nanoparticle surfaces. Normally, more hydrophilic nanoparticle surfaces are known to decrease protein adsorption, but the high negative surface charge-density of PLGA-NPs facilitated electrostatic interactions with the positively charged cytochrome C. Interestingly, it was observed that cytochrome $C$ was able to overcome repulsive coloumbic forces and adsorb onto similar charged surfaces like Eudragit E100 (EU-NPs). Therefore, it is evident that a fine balance between electrostatic and hydrophobic interactions governs the adsorption process of cytochrome C to nanoparticles [30].

It appears that the positive surface charge of stearylamine-PEG-PLA NPs resulted in increased pulmonary side effects along with a transient systemic toxicity mainly on white blood cells. Therefore, these cationic NPs are not recommended for repeated local pulmonary instillation. The overall results suggest that anionic PEG-PLA NPs can be considered as drug carriers for local delivery following repeated instillations to the lungs and merit further investigation [31].

\section{Effect of ZP on pharmacokinetics}

Particle size and surface charge of nano particles regulate the biodistribution and pharmacokinetic properties of the nanoparticles in the body. Altering parameters such as size, conformation and charge can have profound effect on how a nanoparticle acts with and behave in biological environment [32]. Various investigations showed that negatively charged nanoemulsions are cleared faster as well as higher reticuloendothelal uptake than neutral or positively charged nanoemulsions [32]. Other investigations showed that increasing the liposome diameter and adding appositive charge on the liposomes showed higher liposomes clearance from injection site compared with smaller sized and neutral liposomes, respectively, after $2 \mathrm{~h}[16,33]$.

The protein adsorption and cellular uptake of cerium oxide nanoparticles with positive and/or negative surface charge was studied in previous literatures. The comparison of BSA adsorption and nanoparticles ZP clearly showed that positive ZP for nanoceria particles favours the protein adsorption. The negatively charged protein did not significantly adsorb protein [34].

\section{EFFECT OF ZP ON THE PHARMACEUTICAL PROPERTIES OF NANO-DELIVERY SYSTEMS}

\section{Effect of ZP on controlled drug release:}

The release behaviour of drugs in vitro can be controlled by regulating the dissolubility, $\mathrm{pH}$ and Zeta potential of the material [23]. For example, chitosan showed that it could interact with 
negatively charged (acidic) drugs when incorporated into films and this might affect drug release characteristics as well as the physicochemical property of the drug and polymer [35, 36]. As another example, direct interaction between doxorubicin (DOX) and poly (ethylene oxide)-b-poly (methacrylic acid) (PEOb-PMA) block copolymer results in the formation of complexes. The hydrophobic interactions between the anthracycline residues of DOX provide for additional stabilization of the complex. Alternatively, at lower loading, there are more negative charged groups available in the micelles and DOX can be better retained by the electrostatic interactions. Furthermore, faster release of DOX was also observed for the DOXloaded micelles with high density of cross-links $(70 \%)$, which may have more peripheral drug localization and hence faster release [36]. In another study, layer by layer (LbL) coating technique which produces nanoparticle drug delivery systems with improved biocompatibility and sustained or targeted release of drug was used based on sequential electrostatic layer-bylayer (LbL) adsorption of oppositely charged polyelectrolytes (PEs) on a charged substrate produces ultra-thin PE multilayers with controlled properties [37].

\section{Effect of ZP on drug loading efficiency}

The surface charge of the particles and binding type between the drug and nanoparticles are the important parameters that determine the rate of desorption of the drug in the nanoparticles and drug loading efficiency. The zeta potential also can be used to determine whether a charged active material is encapsulated within the center of the nanoparticle or on the surface [38-39]. Cationic charge on liposomes has also been shown to reduce their circulating half life in blood and to affect their biodistribution between the tumor microvasculature and interstitium without impacting overall tumor uptake [40]. Electrostatic interaction between charged block copolymers and oppositely charged macromolecules has allowed the formation of core-shell nanoparticles, which are termed "polyion complex micelles" (PIC) and in this way loading efficiency will be improved $[41,42]$. By measurement of the ZP of the drug or substrate, one can determine if the drug is shielded or not by the nano particles because if the drug is shielded by the nanoparticles ZP will be close to nanoparticle ZP or zero ZP [43]. As an example, the adsorption of tetanus toxoid as a cationic antigen at the negatively charged surface of blank nanoparticles composed of sulfobutylpoly(vinyl alcohol)-g-poly(lactic-co-glycolic acid) copolymers with a core-corona structure showed that the nanoparticle surface properties dictated the adsorption efficiency of tetanus toxoid onto the nanoparticles, and as expected, the maximal loading rate was achieved at the highest degrees of sulfobutyl substitution, which implies that the nature of the tetanus toxoid-nanoparticle interactions was primarily electrostatic [44].

The effect of drug loading on ZP of liposomes was studied through an isothermal titration colorimetry method. The results showed that the addition of drugs (alprenolol, labetalol, propranolol and tetracaien) have no significant effect on the liposome ZP and the ZP remained practically constant. It was discussed that the most probable explanation for the phenomenon is that because of high salt concentration in the medium, the degree of ion binding of sodium ions to the liposome membrane is quite high and they are just replaced by the added drug molecules. Thus the changes in the surface charge of liposmes and hence their effect on the ZP remains minimal during the titration [45]. Nanogels with lower ZP showed higher siRNA amounts [12]. Increasing flurbiprofen loading led to higher ZP, most likely due to the location of the drug molecules onto the NLC surface contributing to their negative charge. The analysis showed that the drug is encapsulated in the polymer matrix rather than physically adsorbed on the nanoparticle surface, and the drug also maintains its crystalline nature inside the matrix. In addition, the entrapment efficiency of calcitonin was observed to increase by increasing the negative zeta potential of the particles, which reflects an ionic interaction between the positively charged peptide drug and the NPs [45].

\section{Effect of ZP on stability of nanoparticle dispersion}

ZP is an indicator of the stability of NP suspensions. A higher electric charge on the surface of the NPs will prevent aggregation of the NPs in buffer solution because of the strong repellent forces among particles [46-47]. Tween 80 also provides a steric stability for maintaining the stability of single layer nanoemultion (SLN) [48-49]. As a rule of thumb, absolute ZP values above $30 \mathrm{mV}$ provide good stability [50-51] and above $60 \mathrm{mV}$ excellent stability. About $20 \mathrm{mV}$ provide only short term stability, values in the range $-5 \mathrm{mV}$ to $+5 \mathrm{mV}$ indicate fast aggregation. This is valid for low molecular weight surfactants and pure electric stabilization, but not for higher or large molecular weight stabilizers, which act mainly by steric stabilisation. In this case ZP values of only $20 \mathrm{mV}$ or much lower can provide 
sufficient stabilization. It has to be kept in mind that adsorbed layers of polymers/large molecules shift the plane of shear to a farer distance from the particle surface. This leads to a reduction of the measured zeta potential. That means even in case of highly charged particle surfaces, a relatively low $Z P$ will be measured. Despite the low ZP, the suspensions are stable [52].

In the case of suspensions, re-suspension of particles from powder is extremely complex. Several factors can influence this process, particularly size, form, electrostatic particle charge and ambient humidity [21]. The stability of emulsion and colloids, according to DLVO electrostatic theory, is a balance between the attractive van der Waals' forces and the electrical repulsion because of the net surface charge. If the zeta potential falls below a certain level, the emulsion droplets or colloids will aggregate as a result of the attractive forces. Conversely, a high zeta potential (either positive or negative), typically more than $30 \mathrm{mV}$, maintains a stable system [29-30].

Colloidal stability in biological environments can be a challenging issue in clinical application of any nanoparticle-based constructs due to the large surface area to volume aspect ratio of nanoscale materials. Charged cationic nanoparticles, in particular, often display poor stability in cell culture conditions, as they tend to adsorb proteins from the biological environment through electrostatic interaction, causing fouling or precipitation.

Zeta potential is an important physicochemical parameter that influences the stability of nanosuspensions. Extremely positive or negative zeta potential values cause larger repulsive forces, whereas repulsion between particles with similar electric charge prevents aggregation of the particles and thus ensures easy redispersion $[34,35]$. In the case of a combined electrostatic and steric stabilization, a minimum zeta potential of $\pm 20 \mathrm{mV}$ is desirable.

\section{Application of ZP in mucoadhesive systems}

Positive zp may give rise the strong electrostatic interaction with mucus or negatively charged mucosal surface. So we can use positively charged nano particles such as chitosan coated nano particles for mucoadhesive drug delivery systems as their positively charged surface can be in favour of adhesion to the cell mucosa which are normally negatively charged [53].

\section{COMPARISION OF POSITIVE AND NEGATIVE ZP IN NANO-DRUG DELIVERY SYSTEMS}

As we said in other parts, positively charged nanoparticles has some advantages in targeted drug delivery because of negatively cell surface but investigation on liposomes showed that both positive and negative charges are known to enhance the delivery of liposomes to cells through adsorptive endocytosis and the half-life clearance of liposomes from the blood can range from minute to hours and the distribution to organs can be controlled in part by altering the physical properties such as size, charge and fluidity. However, positively charged lipids are not approved by FDA for clinical use [2]. Because liposomes or nanoparticles ZP is an important factor for their cytotoxicity and in some cases cationic charge is claimed to enhance cytotoxicity [54-56]. Positive charge posses a membrane destabilizing and concomitantly destructive effect resulting from an interaction of positive charge and negative charge of membrane [57]. The main factors influencing cytotoxicity of positively charged nanoparticles are said to be: 1) molecular weight, 2) charge density and type of cationic functionality, 3) strudture and sequence, 4) conformational flexibility. Structure toxicity relationship could be visualized in a plot of logarithemic IC50 values versus the molecular weight/charge ratio. A linear relationship can be observed [55]. Investigations on dendrimers showed that positively charged amine and guanidine groups, demonstrated dose- and timedependent hemolytic activity [54].

In the case of negatively charged nanoparticles, investigations of liposomes showed that negatively charged liposomes does not show good accumulation in the lymph nodes $(1 / 2 \%$ of the injected dose) in comparision with positively charged (up to $3 / 6 \%$ of the injected dose), and also negatively ones showed lower drug entrapment [58], and some degree of drug release during storage [3], but the negatively charged nano particles are cleared more slowly from the blood compared to positively charged nanoparticles following IV administration to rat, and remained in blood stream for longer time [59].

Isoelectric point values of positively charged nanoparticles above 10 involved very rapid clearance from the blood stream [60]. Also, negatively charged had lower cytotoxicity compared with positively one and approved by FDA. As one can see, both positive and negative zeta potential have their benefits. Therefore, the surface charge density of nano particles should 
be optimized for minimal toxicity and effective intracellular delivery of encapsulated drug.

\section{CONCLUDING REMARK}

Charge modification of nano-systems offer an opportunity for prolonging the blood circulation time of drugs, enhancing the possibility of its interaction with target cells of interest, and changing the pharmaceutical properties of nanosystems.

\section{REFERENCES}

1. Pan J, Shen Feng S. Targeting and imaging cancer cells by Folate-decorated, quantum dots (QDs)- loaded nanoparticles of biodegradable polymers. Biomaterials 2009; 30: 1176-1183.

2. Makhlof A, Werle M, Tozuka Y, Takeuchi H. A mucoadhesive nanoparticulate system for the simultaneous delivery of macromolecules and permeation enhancers to the intestinal mucosa. $\mathrm{J}$. Control. Release 2011; 149: 81-88.

3. Quaglia F, Ostacolo L, Mazzaglia A, Villari V, Zaccaria $D$, Sciortino MT. The intracellular effects of nonionic amphiphilic cyclodextrin nanoparticles in the delivery of anticancer drugs. Biomaterials 2009; 30: 374-382.

4. Feng S, Ruanb G, Lic Q. Fabrication and characterizations of a novel drug deliverydevice liposomes-in microsphere (LIM). Biomaterials 2004; 25: 5181-5189.

5. Zaru M, Manca M, Fadda AM, Antimisiaris SG. Chitosan-coated liposomes for delivery to lungs by nebulisation. Colloid Surfaces B 2009; 71: 88-95.

6. Qi L, Xu Z, Jiang X, Li Y, Wang M. Cytotoxic activities of chitosan nanoparticles and copper-loaded nanoparticles Bioorgan. Med. Chem. Lett. 2005; 15:1397-1399.

7. Bawarski WE, Chidlowsky E, Bharali DJ, Mousa SA. Emerging nanopharmaceuticals. NanomedNanotechnol 2008; 4: 273-282.

8. Unger F, Wlittmar M, Kissel T. Branched polyesters based on poly[vinyl-3-(dialkylamino)alkylcarbamate-co-vinyl acetate-co-vinyl alcohol]-graftpoly(d,l-lactide-co-glycolide) : Effects of polymer structure on cytotoxicity. Biomaterials 2007; 28: 1610-1619.

9. Barratt GM. Therapeutic applications of colloidal drug carriers. Pharm. Sci. Technol. Today 2000; 3: 163171.

10. Wang J, Feng S, Wang S, Chen Z. Evaluation of cationic nanoparticles of biodegradable copolymers as siRNA delivery system for hepatitis B treatment', Int. J. Pharmaceut. 2010; 400: 194-200.

11. Lee SY, Huh MS, Lee S, Lee SJ, Chung H, Park JH, Oh YK, Choi K, Kim K, Kwon IC. Stability and cellular uptake of polymerized siRNA (polysiRNA)/polyethylenimine (PEI) complexes for efficient gene silencing. J. Control. Release 2010; 141: 339-346.

12. Raemdonck K, Naeye B, Høgset A, Demeester J, De Smedt SC. Prolonged gene silencing by combining SiRNA nanogels and photochemical internalization. J. Control. Release 2010; 145: 281-288.

13. Lee $A L$, Wang $Y$, Cheng HY, Pervaiz S, Yang YY. The co-delivery of paclitaxel and Herceptin using cationic micellar nanoparticles. Biomaterials 2009; 30: 919-927.

14. Sarmento $B$, Mazzaglia $D$, Bonferroni MC, Neto $A P$, Monteiro MC, Seabra V. Effect of chitosan coating in overcoming the phagocytosis of insulin loaded solid lipid nanoparticles by mononuclear phagocyte system', Carbohyd. Polym 2011; 84: 919-928.

15. David Needham, Ana Ponce. Nanoscale drug delivery vehicles for Solid tumors, Nanotechnology for cancer therapy, CRC Press, Taylor \& Francis; 2002; $p 677$.

16. He M, Zhao Z, Yin L, Tang C, Yin C. Hyaluronic acid coated poly(butyl cyanoacrylate) nanoparticles as anticancer drug carriers. Int. J. Pharm., 2009; 373: 165-173

17. He M, Hua Y, Yin L, Tang C, Yin C. Effects of particle size and surface charge on cellular uptake and biodistribution of polymeric nanoparticles. Biomaterials 2010; 31: 3657-3666.

18. Rigotti A, Acton SL, Krieger M. The class $B$ scavenger receptors $S R-B I$ and $C D 36$ are receptors for anionic phospholipids. J. Biol. Chem. 1995; 270: 16221- 16224

19. Arnida $M$, Janát-Amsbury MM, Ray A, Peterson CM, Ghandehari $H$. Geometry and surface characteristics of gold nanoparticles influence their biodistribution and uptake by macrophages. Eur. J. Pharm. Biopharm. 2011; 77: 417-423.

20. Obata Y, Tajima S, Takeoka S. Evaluation of $\mathrm{pH}$ responsive liposomes containing amino acid-based zwitterionic lipids for improving intracellular drug delivery in vitro and in vivo. J. Control. Release 2010; 142: 267-276. 28 L1

21. Byrne JD, Betancourt T, Brannon-Peppas L. Active targeting schemes for nanoparticle systems in cancer therapeutics. Adv. Drug Deliver. Rev. 2008; 60: 1615-1626.

22. Csaba N, Garcia-Fuentes M, Alonso MJ. Nanoparticles for nasal vaccination. Adv. Drug Deliver. Rev. 2009; 61: 140-157.

23. Hagigit $T$, Abdulrazik $M$, Orucov $F$, Valamanesh $F$, Lambert M, Lambert G, Behar-Cohen F, Benita S. Topical and intravitreous administration of cationic nanoemulsions to deliver antisense oligonucleotides directed towards VEGF KDR receptors to the eye. J. Control. Release 2010; 145: 297-305.

24. Luoa Q, Zhao J, Zhanga X, Pan W. Nanostructured lipid carrier (NLC) coated with Chitosan Oligosaccharides and its potential use in ocular drug delivery system. Int. J. Pharmaceut. 2011; 403: 185-191.

25. Das S, Suresh PK. Nanosuspension: a new vehicle for the improvement of the delivery of drugs to the ocular surface. Application to amphotericin $B$. Nanomed-Nanotechnol., 2011; 7: 242-

26. Jain JK, Pathana SA, Akhtera $S$, Jayabalanb $N$, Talegaonkara S, Khara RK, Ahmada FJ. Microscopic and spectroscopic evaluation of novel PLGA-chitosan Nanoplexes as an ocular delivery system. Colloid. Surface. B. 2011; 82: 397-403.

27. Khdair A, Chen D, Patil Y, Ma L, Dou P, Shekhar M, Panyam J. Nanoparticle-mediated combination chemotherapy and photodynamic therapy overcomes tumor drug resistance. J. Control. Release 2010; 141: 137-144.

28. Prokop A, Kozlov E, Carlesso J, Davidson M. Hydrogelbased colloidal polymeric system for protein and drug delivery: physical and chemical characterization permeability control and applications. Adv. Polymer res. 2002; 160: 119173.

29. Prokop A, Kozlov E, Carlesso J, Davidson M. Hydrogelbased colloidal polymeric system for protein and drug delivery: physical and chemical characterization permeability control and applications. Adv. Polymer res. 2002; 160: 119173. 
30. Beck-Broichsitter M, Ruppert, C, Schmehl T, Guenther A, Betz T, Bakowsky $U$, Seeger W, Kissel $T$, Gessler T. Biophysical investigation of pulmonary surfactant surface properties upon contact with polymeric nanoparticles in vitro. NanomedNanotechnol 2011; 7: 341-350.

31. Harush-Frenkel O, Bivas-Benita $M$, Nassar T, Springer C, Sherman Y, Avital A, Altschuler Y, Borlak J, Benita S. A safety and tolerability study of differently-charged nanoparticles for local pulmonary drug delivery. Toxicol. Appl. Pharm. (2010) 246; 83-90.

32. Khan MK. Nigavekar SS, Minc LD, Kariapper MS, Nair $B M$, Leniak WG, Balogh LP. In vivo biodistribution of dendrimers and dendrimer nanocomposites -. implications for cancer imaging and therapy. Technol. Cancer Res. Treat. 2005; 4: 603-613.

33. Cal C, Bakowsky U, Rytting E, Scharper AK, Kissel T. Charged nanoparticles as protein delivery systems: A feasibility study using lysozyme as model protein. Eur. J. Pharm. Biopharm. 2008; 69: 31-42.

34. Patila S, Sandberg A, Heckert E, Self W, Sea S. Protein adsorption and cellular uptake of cerium oxide nanoparticles as a function of zeta potential. Biomaterials 2007; 28: 4600-4607.

35. Puttipipatkhachorn S, Nunthanid J, Yamamotoc K, Peck GE. Drug physical state and drug-polymer interaction on drug release from chitosan matrix films. J. Control. Release 2001; 75: 143-153.

36. Kim JO, Kabanov AV, Bronich TK. Polymer micelles with cross-linked polyanion core for delivery of a cationic drug doxorubicin. J. Control. Release 2009; 138: 197-204.

37. Hirsjärvi S, Peltonen L, Hirvonen J. Layer-by-layer polyelectrolyte coating of low molecular weight poly (lactic acid) nanoparticles. Colloid. Surface. B, 2006; 49: 93-99.

38. Honary $S$, Jahanshahi $M$, Golbayani $P$, Ebrahimi $P$. Ghajar K. Doxorubicin- loaded albumin nanoparticles: formulation and Characterization. $\mathrm{J}$. Nanosci. Nanotechno. 2010; 10: 7752-7757.

39. Honary $S$, Ebrahimi $P$, Tabbakhian M, Zahir F. Formulation and characterization of Doxorubicin nanovesicles. J. Vac. Sci. Technol. B, 2009; 27: 1573-1577.

40. Nishiyama N, Kataok K. Current state, achievements, and future prospects of polymeric micelles as nanocarriers for drug and gene delivery. Pharmacol. Therapeut. 2006; 112: 630-648.

41. Kawano K, Takayama K, Nagai $T$, Maitani $Y$. Preparation and pharmacokinetics of pirarubicin loaded dehydration-rehydration vesicles. Int. J. Pharm. 2003; 73-79.

42. Lee ES, Gao Z, Bae Y. Recent progress in tumor pH targeting nanotechnology. J. Control. Release 2008; 132: 164-170.

43. Csaba N, Garcia-Fuentes M, José Alonso M. The performance of nanocarriers for transmucosal drug delivery. Expert Opin. Drug Del. 2006; 3: 463-478.

44. Jung T, Kamm W, Breitenbach A, Hungerer K, Hundt E, Kissel T. Tetanus Toxoid Loaded Nanoparticles from Sulfobutylated Poly(Vinyl Alcohol)-GraftPoly(Lactide-co-Glycolide): Evaluation of Antibody Response After Oral and Nasal Application in Mice. Pharm. Res. 2001; 3: 352-360.
45. J Harting SM, Greene RR, Dikov MM, Prokop A, Davidson JM. Multifunctional nanoparticulate polyelectrolyte complexes. Pharm. Res. 2007; 24: 2353-2369.

46. Sawant K, Dodiya S. Recent Advances and Patents on Solid Lipid Nanoparticles. Recent Pat. Drug Deliv. Formul. 2008; 2: 120-135.

47. Kedar U, Phutane P, Shidhaye S, Kadam V. Advances in polymeric micelles for drug delivery and tumor targeting' Nanomed- Nanotechnol. 2010; 6: 714729.

48. Lambert O, Benoit JP, Pitard B. Encapsulation of DNA molecules within biomimetic lipid nanocapsules. Biomaterials 2009; 30: 3197-3204.

49. Hoeller S, Sperge, A, Valenta C. Lecithin based nanoemulsions: A comparative study of the influence of non-ionic surfactants and the cationic phytosphingosine on physicochemical behaviour and skin permeation. Int. J. Pharm. 2009; 370: 181-186.

50. Wissinga SA, Kayserb O, Muller RH. Solid lipid nanoparticles for parenteral drug delivery. Adv. Drug Deliver. Rev. 2004; 56: 1257- 1272.

51. Jacobs C, Kayser O, Müller R.H. Nanosuspensions as a new approach for the formulation for the poorly soluble drug tarazepide. Int. J. Pharm.2000; 196: 161-164.

52. Quaglia F, Ostacolo L, Mazzaglia A, Villari V, Zaccaria $D$, Sciortino MT. The intracellular effects of nonionic amphiphilic cyclodextrin nanoparticles in the delivery of anticancer drugs. Biomaterials 2009; 30: 374-382.

53. Makhlof A, Werle M, Tozuka Y, Takeuchi H. A mucoadhesive nanoparticulate system for the simultaneous delivery of macromolecules and permeation enhancers to the intestinal mucosa. J. Control. Release 2011; 149: 81-88.

54. Liu J, Hu W, Chena $H$, Nib Q, Xua $H$, Yang X. Isotretinoin-loaded solid lipid nanoparticles with skin mtargeting for topical delivery. Int. J. Pharm. 2007; 328: 191-195.

55. Muller RH, Jacobs C, Kayser O. Nanosuspensions as particulate drug formulations in therapy Rationale for development and what we can expect for the future. Adv. Drug Deliver. Rev. 2001; 47: 3-19.

56. Feng S, Ruanb G, Lic Q. Fabrication and characterizations of a novel drug deliverydevice liposomes-in microsphere (LIM). Biomaterials 2004; 25: 5181-5189.

57. Qi L, Xu Z, Jiang X, Li Y, Wang M. Cytotoxic activities of chitosan nanoparticles and copper-loaded nanoparticles Bioorgan. Med. Chem. Lett. 2005; 15:1397-1399.

58. Bawarski WE, Chidlowsky E, Bharali DJ, Mousa SA. Emerging nanopharmaceuticals. NanomedNanotechnol 2008; 4: 273-282.

59. Unger F, Wlittmar M, Kissel T. Branched polyesters based on poly[vinyl-3(dialkylamino)alkylcarbamate-co-vinyl acetate-covinyl alcoholl-graft-poly (d,I-lactide-co-glycolide) : Effects of polymer structure on cytotoxicity. Biomaterials 2007; 28: 1610-1619.

60. Barratt GM. Therapeutic applications of colloidal drug carriers. Pharm. Sci. Technol. Today 2000; 3: 163171. 